\title{
CARATHÉODORY-TYPE RESULTS FOR THE SUMS AND UNIONS OF CONVEX SETS
}

\author{
JIM LAWRENCE AND VALERIU SOLTAN
}

\begin{abstract}
This paper contains several Carathéodorytype results on extreme representations of sums and unions of finitely many closed convex sets or polyhedra in $\mathbf{R}^{n}$ in terms of their faces.
\end{abstract}

1. Introduction and main results. A well-known result of convex geometry states that any point $z$ of a compact convex set $K \subset \mathbf{R}^{n}$ can be expressed as a convex combination of $n+1$ or fewer extreme points of $K$ (this fact is an immediate consequence of Carathéodory's $[\mathbf{2}]$ and Minkowski's [7, page 160] theorems). Similarly, if $K$ is a line-free closed convex set in $\mathbf{R}^{n}$, then $z$ is a convex combination of $n+1$ or fewer points such that each of the points is either extreme or belongs to an extreme ray of $K$ (see Klee [5]). Simple examples show that the number $n+1$ cannot be decreased in this context. If it is desirable to express $z$ as a convex combination of fewer than $n+1$ points from $K$, then, instead of extreme points or rays, one can consider faces of $K$. Our goal here is to study such extreme representations combined with the operations of addition and union of convex sets.

In what follows, relbd $K$ and relint $K$ stand for the relative boundary and relative interior of a closed convex set $K \subset \mathbf{R}^{n}$, and ext $K$ and $\exp K$ denote, respectively, the set of extreme and exposed points of $K$. A convex set $K \subset \mathbf{R}^{n}$ is line-free if it contains no line of $\mathbf{R}^{n}$. We recall that an (extreme) face of a convex set $K \subset \mathbf{R}^{n}$ is a convex subset $F \subset K$ such that points $x, y \in K$ lie in $F$ provided $(1-\lambda) x+\lambda y \in F$ for a suitable scalar $0<\lambda<1$. Extreme points and extreme rays are, respectively, zero-dimensional and one-dimensional faces. It is easy to see that each proper (i.e., distinct from $K$ ) face of $K$ is closed and lies in relbd $K$. Furthermore, for any point $x \in K$, there is a unique face $F$

2010 AMS Mathematics subject classification. Primary 52A20.

Keywords and phrases. Convex, set, body, polytope, polyhedron, Carathéodory, sum, union.

Received by the editors on March 15, 2011.

DOI:10.1216/RMJ-2013-43-5-1675 Copyright (C)2013 Rocky Mountain Mathematics Consortium 
of $K$ with the property $x \in \operatorname{relint} F$ (see, e.g., Schneider [9] for general references on convex sets).

Theorem 1. Let $K_{1}, \ldots, K_{r}$ be nonempty line-free closed convex sets in $\mathbf{R}^{n}$. For any point $z \in K_{1}+\cdots+K_{r}$, there are nonempty faces $F_{i}$ of $K_{i}, i=1, \ldots, r$, such that

$$
z \in F_{1}+\cdots+F_{r} \quad \text { and } \quad \operatorname{dim} F_{1}+\cdots+\operatorname{dim} F_{r} \leq n .
$$

The proof of this theorem uses a lifting technique introduced (for the case of convex polyhedra) by Walkup and Wets [13]. If the sum $K_{1}+$ $\cdots+K_{r}$ in Theorem 1 is direct, then its assertion becomes a particular case of a statement on the Carathéodory number of the Cartesian product of convexity spaces (see [10] and [11, Theorem 8.18]). Also, Theorem 1 can be formulated in terms of partition of $K_{1}+\cdots+K_{r}$ into sums of relative interiors of suitably chosen faces $F_{i} \subset K_{i}$ (in the spirit of $[6])$.

If the number $r$ in Theorem 1 is greater than $n$, then at least $r-n$ of the faces $F_{i}$ are singletons. This argument allows the refinement of the Shapley-Folkman lemma (see Starr [12]), which states that, for nonempty compact sets $X_{1}, \ldots, X_{r} \subset \mathbf{R}^{n}$ and a point $z \in \operatorname{conv}\left(X_{1}+\right.$ $\left.\cdots+X_{r}\right)$, there is an index set $I \subset\{1, \ldots, r\}$ with $|I| \leq n$ such that

$$
z \in \sum_{i \in I} \operatorname{conv} X_{i}+\sum_{i \notin I} X_{i}
$$

Corollary 1. If $X_{1}, \ldots, X_{r}$ are nonempty sets in $\mathbf{R}^{n}$, then for any point $z$ in $\operatorname{conv}\left(X_{1}+\cdots+X_{r}\right)$, there is an index set $I \subset\{1, \ldots, r\}$ with $|I| \leq n$ and nonempty subsets $Y_{i} \subset X_{i}, i=1, \ldots, r$, such that

$$
\begin{gathered}
z \in \sum_{i \in I} \operatorname{conv} Y_{i}+\sum_{i \notin I} Y_{i}, \\
\sum_{i \in I}\left|Y_{i}\right| \leq n+|I|, \\
\left|Y_{i}\right|=1 \quad \text { for all } i \notin I .
\end{gathered}
$$


Our next theorem deals with unions of convex sets.

Theorem 2. Let $K_{1}, \ldots, K_{r} \subset \mathbf{R}^{n}$ be nonempty line-free closed convex sets. For any point $z \in \operatorname{conv}\left(K_{1} \cup \cdots \cup K_{r}\right)$, there is an index set

$$
I \subset\{1, \ldots, r\} \quad \text { with }|I| \leq n+1
$$

and nonempty faces $F_{i}$ of $K_{i}, i \in I$, such that

$$
z \in \operatorname{conv}\left(\sum_{i \in I} F_{i}\right) \quad \text { and } \sum_{i \in I} \operatorname{dim} F_{i} \leq n .
$$

If, additionally, all $K_{1}, \ldots, K_{r}$ are compact, then the inequality in (3) can be refined to

$$
\sum_{i \in I} \operatorname{dim} F_{i} \leq n+1-|I|
$$

Corollary 2. Let $K \subset \mathbf{R}^{n}$ be a nonempty line-free closed convex set and $r$ a positive integer. For any point $z \in K$, there are nonempty faces $F_{1}, \ldots, F_{s}$ of $K$ with $s \leq \min \{r, n+1\}$ such that

$$
z \in \operatorname{conv}\left(F_{1} \cup \cdots \cup F_{s}\right) \quad \text { and } \quad \operatorname{dim} F_{1}+\cdots+\operatorname{dim} F_{s} \leq n .
$$

If $r>1$, then $F_{1}, \ldots, F_{s}$ can be chosen proper in $K$ such that at least $s-1$ of them are of dimension one or less. If $K$ is compact, then the inequality in (5) can be refined to

$$
\operatorname{dim} F_{1}+\cdots+\operatorname{dim} F_{s} \leq n+1-s
$$

In 1991 Danielyan et al. [3] asked for a sharper version of Corollary 2 (written by us in terms of faces of a convex set in $\mathbf{R}^{n}$ versus $k$-extreme points of a compact convex set in a linear topological space $L$ ). We formulate their question as an open problem below, since its attempted proof in [3] uses the erroneous Property 3 (see [3, page 72]), as shown below. Following [9], a point $x$ of a convex set $M$ is called $k$-extreme 
if it does not belong to the relative interior of a $(k+1)$-dimensional simplex which entirely lies in $M$. Let $E_{k} M$ denote the set of $k$-extreme points of $M$ (thus, $E_{0} M=\operatorname{ext} M$ ). Property 3 from $[\mathbf{3}]$ states that if an $m$-dimensional plane $V$ intersects a compact convex set $M \subset L$, then $E_{i}(M \cap V) \subset E_{i} M$ for all $i=0, \ldots, m$, which is incorrect. Indeed, let $M=\{(x, y, z) \mid 0 \leq x, y, z \leq 1\}$ be the unit cube in $\mathbf{R}^{3}$ and $V \subset \mathbf{R}^{3}$ the plane given by $z=1 / 2$. If $a, b, c, d$ are the vertices of the square $M \cap V$, then $E_{0}(M \cap V)=\{a, b, c, d\} \subset E_{1} M \backslash E_{0} M$. Similarly, $E_{1}(M \cap V) \subset E_{2} M \backslash E_{1} M$.

Problem 1 ([3]). Let $K \subset \mathbf{R}^{n}$ be a nonempty compact convex set and $n_{1}, \ldots, n_{s}$ positive integers with $n_{1}+\cdots+n_{s}=n+1$. Prove that, for any point $z \in K$, there are nonempty faces $F_{1}, \ldots, F_{s}$ of $K$ such that

$$
z \in \operatorname{conv}\left(F_{1} \cup \cdots \cup F_{s}\right) \quad \text { and } \quad \operatorname{dim} F_{i} \leq n_{i}-1 \text { for all } i=1, \ldots, s \text {. }
$$

It is interesting to mention that Motzkin announced in a short abstract [8] (in which conv $\left(F_{1} \cup \cdots \cup F_{k}\right)$ should be read instead of $\left.\operatorname{conv}\left(F_{1} \cap \cdots \cap F_{k}\right)\right)$ that Problem 1 has an affirmative solution when $K$ is a convex polytope in $\mathbf{R}^{n}$ (see also Grünbaum [4, subsection 3.1, Exercise 21]). In this regard, we thank Branko Grünbaum for providing information about Motzkin's abstract. Corollary 3 below confirms Motzkin's statement. We also observe that Problem 1 has an affirmative solution for any compact convex set in $\mathbf{R}^{n}$ if $n \leq 3$.

Two more results deal with intersections of convex polyhedra. In what follows, by a polyhedron we mean the intersection of finitely many closed halfspaces of $\mathbf{R}^{n}$; a polytope is the convex hull of finitely many points.

Lemma 1. Let $P_{1}, \ldots, P_{t} \subset \mathbf{R}^{n}, 1 \leq t \leq n$, be nonempty linefree polyhedral cones with common apex $o$ and $n_{1}, \ldots, n_{t}$ nonnegative integers with $n_{1}+\cdots+n_{t}=n$. For any point $z \in P_{1} \cap \cdots \cap P_{t}$, there are nonempty faces $F_{i}$ of $P_{i}$ such that

$z \in \operatorname{conv}\left(F_{1} \cup \cdots \cup F_{t}\right) \quad$ and $\quad \operatorname{dim} F_{i} \leq n_{i}$ for all $i=1, \ldots, t$. 
Theorem 3. Let $P_{1}, \ldots, P_{s} \subset \mathbf{R}^{n}$ be nonempty polytopes and $n_{1}, \ldots, n_{s}$ positive integers with $n_{1}+\cdots+n_{s}=n+1$. For any point $z \in P_{1} \cap \cdots \cap P_{s}$, there are nonempty faces $F_{i}$ of $P_{i}, i=1, \ldots, s$, such that

$$
z \in \operatorname{conv}\left(F_{1} \cup \cdots \cup F_{s}\right) \quad \text { and } \quad \operatorname{dim} F_{i} \leq n_{i}-1 \text { for all } i=1, \ldots, s .
$$

With $s=n+1$ and $n_{i}=1$ for all $i=1, \ldots, n+1$, Theorem 3 gives a new way to prove "the colorful version" of Carathéodory's theorem due to Bárány [1], which states that, given nonempty sets $X_{1}, \ldots, X_{n+1} \subset \mathbf{R}^{n}$ and a point $z \in \operatorname{conv} X_{1} \cap \cdots \cap \operatorname{conv} X_{n+1}$, there are points $v_{i} \in X_{i}, i=1, \ldots, n+1$, such that $z \in \operatorname{conv}\left\{v_{1}, \ldots, v_{n+1}\right\}$.

Corollary 3. Let $P \subset \mathbf{R}^{n}$ be a nonempty polytope and $n_{1}, \ldots, n_{s}$ positive integers with $n_{1}+\cdots+n_{s}=n+1$. For any point $z \in P$, there are nonempty faces $F_{1}, \ldots, F_{s}$ of $P$ such that

$z \in \operatorname{conv}\left(F_{1} \cup \cdots \cup F_{s}\right) \quad$ and $\quad \operatorname{dim} F_{i} \leq n_{i}-1$ for all $i=1, \ldots, s$.

In a standard way, both Corollaries 2 and 3 can be slightly refined by replacing $n$ with $\operatorname{dim} K$ and $\operatorname{dim} P$, respectively.

\section{Proofs.}

Proof of Theorem 1. Choose an exposed point $v_{i}$ of $K_{i}$ (it exists because $K_{i}$ is line-free) and denote by $H_{i}$ a hyperplane with the property $K_{i} \cap H_{i}=\left\{v_{i}\right\}, i=1, \ldots, r$. We can write $H_{i}=\{x \in$ $\left.\mathbf{R}^{n} \mid \psi_{i}(x)=\alpha_{i}\right\}$, where $\psi_{i}$ is a nonzero linear functional on $\mathbf{R}^{n}$ and $\alpha_{i}$ is a scalar. Without loss of generality, suppose that $\alpha_{i}$ is the minimum value of $\psi_{i}$ on $K_{i}$.

We observe that, for any scalar $c \in \mathbf{R}$, the set $K_{i}(c)=\left\{x \in K_{i} \mid\right.$ $\left.\psi_{i}(x) \leq c\right\}$ is compact. Indeed, assume for a moment that $K_{i}(c)$ is not compact for a certain choice of $c \in \mathbf{R}$. Then $K_{i}(c)$ contains a halfline $h=[u, w)$. Since $\psi_{i}$ attains a minimum on $K_{i}$, the halfline $h$ must be parallel to $H_{i}$. In this case, $\psi_{i}(x)=\alpha_{i}$ for all $x \in h^{\prime}=\left(v_{i}-u\right)+h$, implying that $h^{\prime}$ lies in $K_{i} \cap H_{i}$, in contradiction with the choice of $H_{i}$. 
For each $\psi_{i}, i=1, \ldots, r$, choose a basis $\psi_{i}, \gamma_{2}^{(i)}, \ldots, \gamma_{n}^{(i)}$ of linear functionals for the conjugate space $\left(\mathbf{R}^{n}\right)^{\prime}$. Consider on $\mathbf{R}^{n}$ the nonsingular linear transformations

$$
f_{i}(x)=\left(\psi_{i}(x), \gamma_{2}^{(i)}(x), \ldots, \gamma_{n}^{(i)}(x)\right), \quad i=1, \ldots, r .
$$

Given a vector $\bar{x}=\left(x_{1}, \ldots, x_{r}\right) \in\left(\mathbf{R}^{n}\right)^{r}$, put

$$
f(\bar{x})=\left(f_{1}\left(x_{1}\right), \ldots, f_{r}\left(x_{r}\right)\right) .
$$

Clearly, $f$ is a nonsingular linear transformation on $\left(\mathbf{R}^{n}\right)^{r}$. Let

$$
\bar{v}=\left(v_{1}, \ldots, v_{r}\right) \quad \text { and } \quad K=K_{1} \times \cdots \times K_{r} .
$$

From the choice of $\psi_{1}, \ldots, \psi_{r}$ it follows that $f(\bar{v})$ is the unique lexicographically minimal point of $f(K)$. (Recall that a point $x=$ $\left(x_{1}, \ldots, x_{m}\right) \in \mathbf{R}^{m}$ is lexicographically smaller than a point $x^{\prime}=$ $\left(x_{1}^{\prime}, \ldots, x_{m}^{\prime}\right) \in \mathbf{R}^{m}$, provided $x \neq x^{\prime}$ and $x_{i}<x_{i}^{\prime}$ for the smallest index $i=1, \ldots, m$ with $x_{i} \neq x_{i}^{\prime}$.) Moreover, for any nonempty closed set $X \subset K$, the set $f(X)$ contains a unique lexicographically minimal point, $\bar{u}$. The existence of $\bar{u}$ immediately follows from continuity of $f$ and compactness of $K(c)=K_{1}(c) \times \cdots \times K_{r}(c)$ for any choice of $c \in \mathbf{R}$. Since $f$ is nonsingular, the set $f^{-1}(\bar{u})$ is a singleton.

Consider the linear subspace

$$
L=\left\{\bar{x}=\left(x_{1}, \ldots, x_{r}\right) \in\left(\mathbf{R}^{n}\right)^{r} \mid x_{1}+\cdots+x_{r}=o\right\} .
$$

For any point $\bar{x} \in K$, denote by $\varphi(\bar{x})$ the inverse image of the unique lexicographically minimal point of the set $f(K \cap(\bar{x}+L))$. Clearly, $\varphi(\bar{v})=\bar{v}$ and $\varphi(\bar{x}) \in K \cap(\bar{x}+L)$ whenever $\bar{x} \in K$. Let $B=\varphi(K)$. Since $\varphi(\varphi(\bar{x}))=\varphi(\bar{x})$ for any $\bar{x} \in K$, we have

$$
B=\{\bar{x} \in K \mid \varphi(\bar{x})=\bar{x}\} .
$$

Let $z$ be a given point in $K_{1}+\cdots+K_{r}$. Then $z=z_{1}+\cdots+z_{r}$ for suitable points $z_{i} \in K_{i}, i=1, \ldots, r$. Put

$$
\bar{z}=\left(z_{1}, \ldots, z_{r}\right) \text { and } \varphi(\bar{z})=\left(z_{1}^{\prime}, \ldots, z_{r}^{\prime}\right) .
$$


Since $\varphi(\bar{z}) \in K \cap(\bar{z} \cap L)$, we have $\varphi(\bar{z})=\bar{z}+\bar{x}$ for a suitable point $\bar{x} \in L$, so $z_{1}^{\prime}+\cdots+z_{r}^{\prime}=\left(z_{1}+\cdots+z_{r}\right)+\left(x_{1}+\cdots+x_{r}\right)=\left(z_{1}+\cdots+z_{r}\right)+o=z$.

Denote by $F$ the face of $K$ that contains $\varphi(\bar{z})$ in its relative interior. We state that $F \subset B$. Indeed, assume for a moment the existence of a point $\bar{w} \in F \backslash B$. Then $\varphi(\bar{w}) \neq \bar{w}$, which means that $f(\varphi(\bar{w}))$ is lexicographically smaller that $f(\bar{w})$. Since $\varphi(\bar{z}) \in \operatorname{relint} F$, there is a point $\bar{u} \in F$ such that $\varphi(\bar{z})=(1-\lambda) \bar{u}+\lambda \bar{w}$ for a suitable scalar $0<\lambda<1$. Let $\bar{y}=(1-\lambda) \bar{u}+\lambda \varphi(\bar{w})$. By a convexity argument, $\bar{y} \in K$. Since both $\bar{w}$ and $\varphi(\bar{w})$ lie in $\bar{w}+L$, we have $\varphi(\bar{w})-\bar{w} \in L$. Therefore,

$$
\bar{y}-\varphi(\bar{z})=\lambda(\varphi(\bar{w})-\bar{w}) \in L .
$$

Hence $\bar{y} \in K \cap(\varphi(\bar{z})+L)=K \cap(\bar{z}+L)$. The equalities

$$
f(\bar{y})=(1-\lambda) f(\bar{u})+\lambda f(\varphi(\bar{w})), \quad f(\varphi(\bar{z}))=(1-\lambda) f(\bar{u})+\lambda f(\bar{w})
$$

show that $f(\bar{y})$ is lexicographically smaller than $f(\varphi(\bar{z}))$. This is impossible due to the definition of $\varphi(\bar{z})$. Summing up, $F \subset B$.

We can write $F=F_{1} \times \cdots \times F_{s}$, where $F_{i}$ is a nonempty face of $K_{i}$, $i=1, \ldots, r$. From $\varphi(\bar{z}) \in F$, it follows that $z_{i}^{\prime} \in F_{i}$ for all $i=1, \ldots, r$. Observe that the linear transformation $\pi:\left(\mathbf{R}^{n}\right)^{r} \rightarrow \mathbf{R}^{n}$, defined by

$$
\pi\left(\left(x_{1}, \ldots, x_{r}\right)\right)=x_{1}+\cdots+x_{r},
$$

is one-to-one on $B$. Indeed, let $\bar{x}=\left(x_{1}, \ldots, x_{r}\right)$ and $\bar{x}^{\prime}=\left(x_{1}^{\prime}, \ldots, x_{r}^{\prime}\right)$ be two points in $B$ with $\pi(\bar{x})=\pi\left(\bar{x}^{\prime}\right)$. The equality $x_{1}+\cdots+x_{r}=$ $x_{1}^{\prime}+\cdots+x_{r}^{\prime}$ implies that $\bar{x}+L=\bar{x}^{\prime}+L$. Due to the uniqueness of $\varphi(\bar{x})$ in $K \cap(\bar{x}+L)$, one has $\bar{x}=\varphi(\bar{x})=\varphi\left(\bar{x}^{\prime}\right)=\bar{x}^{\prime}$. This argument and the equalities

$$
F=F_{1} \times \cdots \times F_{r}, \quad \pi(F)=F_{1}+\cdots+F_{r}
$$

show that

$$
\begin{aligned}
\operatorname{dim} F_{1}+\cdots+\operatorname{dim} F_{r} & =\operatorname{dim} F=\operatorname{dim} \pi(F) \\
& =\operatorname{dim}\left(F_{1}+\cdots+F_{r}\right) \leq n,
\end{aligned}
$$


because $F_{1}+\cdots+F_{r}$ is a subset of $\mathbf{R}^{n}$. Finally,

$$
z=z_{1}^{\prime}+\cdots+z_{r}^{\prime} \in F_{1}+\cdots+F_{r}
$$

Proof of Corollary 1. From the inclusion

$$
z \in \operatorname{conv}\left(X_{1}+\cdots+X_{r}\right)=\operatorname{conv} X_{1}+\cdots+\operatorname{conv} X_{r}
$$

it follows that $z$ can be expressed as $z=z_{1}+\cdots+z_{r}$, where $z_{i} \in \operatorname{conv} X_{i}$. By Carathéodory's theorem, there is a finite set $Z_{i} \subset X_{i}$ such that $z_{i} \in \operatorname{conv} Z_{i}$. Consider the convex polytopes $P_{i}=\operatorname{conv} Z_{i}, i=1, \ldots, r$. By Theorem 1, one can find nonempty faces $F_{i}$ of $P_{i}, i=1, \ldots, r$, such that the conditions (1) hold. Put $n_{i}=\operatorname{dim} P_{i}$.

Clearly, $F_{i}=\operatorname{conv} V_{i}$ for a certain subset $V_{i}$ of $Z_{i}$. Again by Carathéodory's theorem, there are subsets $Y_{i} \subset V_{i}$ such that

$$
z_{i} \in \operatorname{conv} Y_{i} \text { and }\left|Y_{i}\right| \leq n_{i}+1 \text { for all } i=1, \ldots, r
$$

The inequality $n_{1}+\cdots+n_{r} \leq n$ implies the existence of an index set $I \subset\{1, \ldots, r\}$ with $|I| \leq n$ such that $n_{i}=0$ for all $i \notin I$. Equivalently, $\left|Y_{i}\right|=1$, or $Y_{i}=\left\{z_{i}\right\}$, when $i \notin I$. Finally,

$$
z=\sum_{i=1}^{r} z_{i} \in \sum_{i \in I} \operatorname{conv} Y_{i}+\sum_{i \notin I} Y_{i}
$$

where

$$
\sum_{i \in I}\left|Y_{i}\right| \leq n+|I| \quad \text { and } \quad\left|Y_{i}\right|=1 \text { for all } i \notin I
$$

Proof of Theorem 2. According to Carathéodory's theorem, there are points

$$
z_{1}, \ldots, z_{s} \in K_{1} \cup \cdots \cup K_{r}, \quad s \leq n+1,
$$

such that $z$ can be written as their convex combination:

$$
z=\alpha_{1} z_{1}+\cdots+\alpha_{s} z_{s}, \quad \alpha_{1}, \ldots, \alpha_{s} \geq 0, \quad \alpha_{1}+\cdots+\alpha_{s}=1 .
$$


Eliminating zero scalars, we may suppose that all $\alpha_{1}, \ldots, \alpha_{s}$ are positive. Furthermore, $z_{1}, \ldots, z_{s}$ can be chosen such that no two of them belong to the same set $K_{i}$. Indeed, if $z_{p}, z_{q} \in K_{i}$, we replace $\alpha_{p} z_{p}+\alpha_{q} z_{q}$ in (7) with $\left(\alpha_{p}+\alpha_{q}\right) z_{p}^{\prime}$, where $z_{p}^{\prime}$ is a convex combination of $z_{p}$ and $z_{q}$ :

$$
z_{p}^{\prime}=\frac{\alpha_{p}}{\alpha_{p}+\alpha_{q}} z_{p}+\frac{\alpha_{q}}{\alpha_{p}+\alpha_{q}} z_{q} \in K_{i} .
$$

As a result, $s \leq r$. Renumbering $z_{1}, \ldots, z_{s}$ and $K_{1}, \ldots, K_{r}$, we assume that $z_{i} \in K_{i}$ for all $i=1, \ldots, s$. Put $I=\{1, \ldots, s\}$.

Consider the convex set $K=K_{1} \times \cdots \times K_{s} \subset\left(\mathbf{R}^{n}\right)^{s}$. Since all $K_{1}, \ldots, K_{s}$ are closed and line-free, $K$ is also closed and line-free. Put

$$
P=\left\{\left(x_{1}, \ldots, x_{s}\right) \in\left(\mathbf{R}^{n}\right)^{s} \mid \alpha_{1} x_{1}+\cdots+\alpha_{s} x_{s}=z\right\} .
$$

With given positive scalars $\alpha_{1}, \ldots, \alpha_{s}$ and vector $z=\left(\eta_{1}, \ldots, \eta_{n}\right) \in$ $\mathbf{R}^{n}$, the equality $\alpha_{1} x_{1}+\cdots+\alpha_{s} x_{s}=z$ can be rewritten as the system of $n$ linear equations in $n s$ scalar variables $x_{i}=\left(\xi_{1}^{(i)}, \ldots, \xi_{n}^{(i)}\right)$, $i=1, \ldots, s$ :

$$
\alpha_{1} \xi_{j}^{(1)}+\alpha_{2} \xi_{j}^{(2)}+\cdots+\alpha_{s} \xi_{j}^{(s)}=\eta_{j}, \quad j=1, \ldots, n .
$$

Because the rank of this system is $n$, we conclude that $P$ is an affine subspace of $\left(\mathbf{R}^{n}\right)^{s}$ of dimension $n(s-1)$. This argument shows that $M=K \cap P$ is a line-free closed convex set of dimension $n(s-1)$ or less. Furthermore, $\left(z_{1}, \ldots, z_{s}\right) \in M$ due to (7).

Since $M$ is line-free, it contains an extreme point, $\bar{w}=\left(w_{1}, \ldots, w_{n}\right)$. Denote by $F$ the (unique) face of $K$ that contains $\bar{w}$ in its relative interior. We can write $F=F_{1} \times \cdots \times F_{s}$, where each $F_{i}$ is a nonempty face of $K_{i}$ such that $w_{i} \in \operatorname{relint} F_{i}, i=1, \ldots, s$. From this and the inclusion $\bar{w} \in M$, it follows that

$$
z=\alpha_{1} w_{1}+\cdots+\alpha_{s} w_{s} \in \operatorname{conv}\left(F_{1} \cup \cdots \cup F_{s}\right) .
$$

It remains to prove the inequality from (3). First, we observe that $F \cap P=\{\bar{w}\}$. Indeed, assume the existence of another point $\bar{u} \in F \cap P$. From $\bar{w} \in \operatorname{relint} F$, it follows that $\bar{w} \in] \bar{u}, \bar{v}[\subset F$ for a suitable point $\bar{v} \in F$. Clearly, $] \bar{u}, \bar{v}[\subset P$, which implies the 
inclusion $\bar{w} \in] \bar{u}, \bar{v}[\subset F \cap P \subset M$. The last is in contradiction with the assumption $\bar{w} \in \operatorname{ext} M$.

Denote by $Q$ the smallest affine subspace of $\left(\mathbf{R}^{n}\right)^{s}$ containing $F$. We state that $P \cap Q=\{\bar{w}\}$. Indeed, assume that $P \cap Q$ contains more than one point. Then $P \cap Q$ contains a line $l$ through $\bar{w}$. Since $\bar{w} \in \operatorname{relint} F$, the intersection $l \cap F$ contains an open line segment $] \bar{a}, \bar{c}[$ with $\bar{w} \in] \bar{a}, \bar{c}[\subset F$. Because $l \subset P$, we have $\bar{w} \in] \bar{a}, \bar{c}[\subset P \cap F \subset M$, which is impossible due to $\bar{w} \in \operatorname{ext} M$.

From $P \cap Q=\{\bar{w}\}$ it follows that $\operatorname{dim} P+\operatorname{dim} Q \leq \operatorname{dim}\left(\mathbf{R}^{n}\right)^{s}=n s$. Hence,

$$
\operatorname{dim} F_{1}+\cdots+\operatorname{dim} F_{s}=\operatorname{dim} F=\operatorname{dim} Q \leq n s-\operatorname{dim} P=n .
$$

Now assume that all $K_{1}, \ldots, K_{r}$ are compact. We identify $\mathbf{R}^{n}$ with the hyperplane

$$
H=\left\{\left(x_{1}, \ldots, x_{n+1}\right) \mid x_{n+1}=1\right\} \subset \mathbf{R}^{n+1} .
$$

Let $K_{i}^{\prime}$ be the convex cone with apex $o$ generated by $K_{i}, i=1, \ldots, r$. Clearly, $K_{1}^{\prime}, \ldots, K_{r}^{\prime}$ are line-free closed convex cones. From the above (with $\mathbf{R}^{n+1}$ instead of $\mathbf{R}^{n}$ ), there is an index set $I \subset\{1, \ldots, r\}$ with $|I| \leq n+2$ and nonempty faces $G_{i}$ of $K_{i}^{\prime}, i \in I$, such that

$$
z \in \operatorname{conv}\left(\bigcup_{i \in I} G_{i}\right) \text { and } \sum_{i \in I} \operatorname{dim} G_{i} \leq n+1 .
$$

Without loss of generality, we may assume that $I=\{1, \ldots, s\}$, where $s \leq \min \{r, n+2\}$.

All faces $G_{1}, \ldots, G_{s}$ are line-free closed convex cones with common apex $o$. We may assume that each $G_{i}$ is distinct from $\{o\}$, since otherwise the removal of the respective index $i$ from $I$, keeps both conditions from (9). Furthermore, $s \leq n+1$, since otherwise $\operatorname{dim} G_{1}+$ $\cdots+\operatorname{dim} G_{s} \geq n+2$.

The inequalities $\operatorname{dim} G_{i} \geq 1, i=1, \ldots, s$, also imply that each $G_{i}$ meets $H$. Put $F_{i}=G_{i} \cap H, i=1, \ldots, s$. Clearly, $\operatorname{dim} F_{i}=\operatorname{dim} G_{i}-1$, resulting in

$$
\operatorname{dim} F_{1}+\cdots+\operatorname{dim} F_{s} \leq \operatorname{dim} G_{1}+\cdots+\operatorname{dim} G_{s}-s \leq n+1-s .
$$


It remains to show that $z \in \operatorname{conv}\left(F_{1} \cup \cdots \cup F_{s}\right)$. Indeed, as in the beginning of the proof of this theorem, the inclusion $z \in \operatorname{conv}\left(G_{1} \cup\right.$ $\cdots \cup G_{s}$ ) yields the existence of points $z_{i} \in G_{i}, i=1, \ldots, s$, such that (7) holds for a suitable choice of scalars $\alpha_{1}, \ldots, \alpha_{s}$. Without loss of generality, we may suppose that all vectors $z_{1}, \ldots, z_{s}$ are distinct from $o$. Therefore, we can choose positive scalars $\beta_{1}, \ldots, \beta_{s}$ such that $w_{i}=\beta_{i} z_{i} \in H$ for all $i=1, \ldots, s$. Since $w_{i} \in G_{i}$, we have $w_{i} \in F_{i}$, $i=1, \ldots, s$, and

$$
z=\left(\alpha_{1} \beta_{1}\right) w_{1}+\cdots+\left(\alpha_{s} \beta_{s}\right) w_{s}, \quad \alpha_{1} \beta_{1}, \ldots, \alpha_{s} \beta_{s}>0 .
$$

The inclusions $z, w_{1}, \ldots, w_{s} \in H$ imply that the $(n+1)$ th coordinate of all points $z, w_{1}, \ldots, w_{s}$ equals 1 . This argument and (10) give $\alpha_{1} \beta_{1}+\cdots+\alpha_{s} \beta_{s}=1$, which shows that $z$ is a convex combination of $w_{1}, \cdots, w_{s}$. Therefore $z \in \operatorname{conv}\left(F_{1} \cup \cdots \cup F_{s}\right)$.

Proof of Corollary 2. The existence of nonempty faces $F_{1}, \ldots, F_{s}$ of $K$, with $s \leq \min \{r, n+1\}$, which satisfy the conditions (2) immediately follows from Theorem 2. Let $r>1$. We intend to prove the second statement of the corollary by induction on $m=\operatorname{dim} K$. Since the case $\operatorname{dim} K \leq 1$ is trivial, we may assume that $m>1$. Because $K$ is line-free, there is a hyperplane $H \subset \mathbf{R}^{n}$ through $z$ such that $K \cap H$ is bounded. Let $u$ be an extreme point of $K \cap H$. It is easy to see that the face $F_{1}$ of $K$ that contains $u$ in its relative interior is either a singleton or a halfline, implying that $\operatorname{dim} F_{1} \leq 1$. If $u=z$, the proof is finished. Let $u \neq z$. The line $(u, z)$ intersects bd $K$ at a point $v \in \operatorname{bd} K$ distinct from $u$. Denote by $F$ the face of $K$ such that $v \in \operatorname{relint} F$. Then $F$ is a line-free closed convex set such that $\operatorname{dim} F \leq n-1$ and $z \in \operatorname{conv}\left(F_{1} \cup F\right)$. In particular, $F$ lies in a hyperplane $G$ of $\mathbf{R}^{n}$, which can be identified with $\mathbf{R}^{n-1}$. By the inductive assumption, there are faces $F_{2}, \ldots, F_{s}, s \leq r$, such that

$$
v \in \operatorname{conv}\left(F_{1} \cup \cdots \cup F_{s}\right), \quad \operatorname{dim} F_{2}+\cdots+\operatorname{dim} F_{s} \leq n-1,
$$

and $\operatorname{dim} F_{i} \leq 1$ for at least $s-2$ of them. Clearly, $F_{1}, \ldots, F_{s}$ satisfy the conditions (5). The last statement of the corollary trivially follows from Theorem 2 .

Proof of Lemma 1. Choose nonempty faces $F_{i} \subset P_{i}$ with $\operatorname{dim} F_{i} \leq n_{i}$ for all $i=1, \ldots, t$ such that the distance $\delta$ from $z$ to the set $F=$ 
conv $\left(F_{1} \cup \cdots \cup F_{t}\right)$ is minimized. All $F_{1}, \ldots, F_{t}$ are convex polyhedral cones with common apex $o$. Therefore, $F$ is either the whole space $\mathbf{R}^{n}$ or a convex polyhedral cone with apex $o$. If $\delta=0$, the proof is finished. Assume that $\delta>0$. Then $z \neq o$ and $F \neq \mathbf{R}^{n}$. Let $v$ be the point of $F$ that is closest to $z$. Then the hyperplane

$$
H=\left\{x \in \mathbf{R}^{n} \mid(z-v) \cdot x=0\right\}
$$

supports $F$ and $v \in H$. Clearly, $o \in H$ because $F$ is a cone with apex $o$. Denote by $H^{+}$the open halfspace bounded by $H$ that contains $z$. Consider the faces $E_{i}=H \cap F_{i}, i=1, \ldots, t$. By the above, $E_{1}, \ldots, E_{t}$ are line-free convex polyhedral cones with common apex $o$ and

$$
v \in H \cap \operatorname{conv}\left(F_{1} \cup \cdots \cup F_{t}\right)=\operatorname{conv}\left(E_{1} \cup \cdots \cup E_{t}\right) .
$$

Theorem 2 (with $H$ instead of $\mathbf{R}^{n}$ ) implies the existence of an index set $I \subset\{1, \ldots, t\}$ and nonempty faces $G_{i}$ of $E_{i}, i \in I$, such that

$$
z \in \operatorname{conv}\left(\bigcup_{i \in I} G_{i}\right) \text { and } \sum_{i \in I} \operatorname{dim} G_{i} \leq n-1
$$

Adding, if necessary, trivial faces $G_{i}=\{o\}$ of $E_{i}$ for all $i \notin I$, we may assume that $I=\{1, \ldots, t\}$. Clearly, $G_{i}$ is a face of $F_{i}$ and $\operatorname{dim} G_{i} \leq \operatorname{dim} F_{i} \leq n_{i}, i=1, \ldots, s$. The inequality

$$
\operatorname{dim} G_{1}+\cdots+\operatorname{dim} G_{t}<n=n_{1}+\cdots+n_{t}
$$

implies that $\operatorname{dim} G_{j}<n_{j}$ for at least one $j=1, \ldots, t$. Since $z \in$ $P_{j} \cap H^{+}$, there is a face $F_{j}^{\prime}$ of $P_{j}$ of dimension at most $n_{j}$ that contains $G_{j}$ and intersects $H^{+}$(otherwise $P_{j}$ would entirely lie in the opposite closed halfspace of $\mathbf{R}^{n}$ bounded by $H$ ). Put $G_{j}^{\prime}=F_{j}^{\prime} \cap H$ and

$$
F^{\prime}=\operatorname{conv}\left(F_{1} \cup \cdots F_{j-1} \cup F_{j}^{\prime} \cup F_{j+1} \cup \cdots \cup F_{t}\right) .
$$

Then $G_{j} \subset G_{j}^{\prime}$, which gives $v \in F^{\prime}$. Taking a point $u \in F_{j}^{\prime} \cap H^{+}$, we can easily find a point $\left.w \in^{\prime}\right] u, v\left[\subset F^{\prime}\right.$ such that $\|w-z\|<\|v-z\|$, in contradiction with the choice of $\delta$. Hence, $\delta$ should be zero, which shows that $F_{1}, \ldots, F_{t}$ satisfies the conclusion of the theorem. 
3. Proof of Theorem 3. Identifying $\mathbf{R}^{n}$ with the hyperplane

$$
H=\left\{\left(x_{1}, \ldots, x_{n+1}\right) \mid x_{n+1}=1\right\} \subset \mathbf{R}^{n+1},
$$

consider the line-free polyhedral cones $Q_{i}=\operatorname{pos} P_{i} \subset \mathbf{R}^{n+1}, i=$ $1, \ldots, s$. Then $z \in Q_{1} \cap \cdots \cap Q_{s}$. By Lemma 1 , there are nonempty faces $G_{i}$ of $Q_{i}$ such that

$z \in \operatorname{conv}\left(G_{1} \cup \cdots \cup G_{s}\right)$ and $\operatorname{dim} G_{i} \leq n_{i}$ for all $i=1, \ldots, s$.

For each $i=1, \ldots, s$, define a set $F_{i}$ as follows:

(i) if $G_{i} \neq\{o\}$, then put $F_{i}=G_{i} \cap H$,

(ii) if $G_{i}=\{o\}$, then choose any extreme point $z_{i}$ of $P_{i}$ and put $F_{i}=\left\{z_{i}\right\}$.

Clearly, $F_{i}$ is a nonempty face of $P_{i}, i=1, \ldots, s$. As in the proof of Theorem 2, one has $z \in \operatorname{conv}\left(F_{1} \cup \cdots \cup F_{s}\right)$. Furthermore, if $I$ denotes the set of all indices $i=1, \ldots s$ for which $\operatorname{dim} G_{i}>0$, then

$$
\operatorname{dim} F_{i}= \begin{cases}\operatorname{dim} G_{i}-1 \leq n_{i}-1 & i \in I, \\ 0 \leq n_{i}-1 & \text { if } i \notin I .\end{cases}
$$

Acknowledgments. The authors thank the anonymous referee for helpful comments on an earlier draft of this article.

\section{REFERENCES}

1. I. Bárány, A generalization of Carathéodory's theorem, Discr. Math. 40 (1982), 141-152.

2. C. Carathéodory, Über den Variabilitätsbereich der Fourierschen Konstanten von positiven harmonischen Funktionen, Rend. Circ. Mat. Palermo 32 (1911), 193-217.

3. È.A. Danielyan, G.S. Movsisyan and K.R. Tatalyan, Generalization of the Carathéodory theorem, Akad. Nauk Armenii Dokl. 92 (1991), 69-75.

4. B. Grünbaum, Convex polytopes, Interscience Publishers, New York, 1967. Second edition: Springer-Verlag, New York, 2003.

5. V.L. Klee, Extremal structure of convex sets, Arch. Math. 8 (1957), 234-240.

6. J. Lawrence and V. Soltan, The intersection of convex transversals is a convex polytope, Beitr. Alg. Geom. 50 (2009), 283-294. 
7. H. Minkowski, Theorie der konvexer Körper, insbesondere Begründung ihres Oberflächenbegriffs, in Gesammelte Abhandlungen, B.G. Teubner, Leipzig, 1911.

8. T. Motzkin, Extensions of the Minkowski-Carathéodory theorem on convex hulls, Abstract 65T-385, Not. Amer. Math. Soc. 12 (1965), 705.

9. R. Schneider, Convex bodies: The Brunn-Minkowski theory, Cambridge University Press, Cambridge, 1993.

10. V. Soltan, Substitution numbers and Caratheodory numbers of the Cartesian product of convexity structures, Ukrain. Geom. Sb. No. 24 (1981), 104-108.

11. - Introduction to the axiomatic theory of convexity, Ştiința, Chişinău, 1984.

12. R.M. Starr, Quasi-equilibria in markets with non-convex preferences, Econometrica 37 (1969), 25-38.

13. D.W. Walkup and R.J.-B. Wets, Lifting projections of convex polyhedra, Pacific J. Math. 28 (1969) 465-475.

Department of Mathematical Sciences, George Mason University, 4400 University Drive, FAirfax, VA 22030

Email address: lawrence@gmu.edu

Department of Mathematical Sciences, George Mason University, 4400 University Drive, Fairfax, VA 22030

Email address: vsoltan@gmu.edu 